\title{
Novel Promises of Nanotechnology for Tissue Regeneration
}

\author{
Abir El-Sadik \\ Anatomy and Embryology, Basic Sciences Department, \\ King Saud Bin Abdulaziz University for Health Sciences, Riyadh \\ Kingdom of Saudi Arabia
}

\section{Introduction}

The term 'nanotechnology' refers to technology that deals with structures and devices of nanometer (10 - 9 meter) size. It involves the design, fabrication and utilization of materials of nanoscale dimensions (Gao \& Xu, 2009). The resulting nanomaterials exhibit chemical, physical and biological properties that can differ significantly from those of bulk material. These products can be categorized into metals, ceramics, polymers or composite materials that have nanoscale features. The limited size of their particles leads to a high surface area to volume ratio, improved solubility, multifunctionality, high electrical and heat conductivity and improved surface catalytic activity. (El-Sadik et al., 2010). All these phenomena allow give nanoparticles to interact with biological systems at cellular and molecular levels. These interactions enhance the biomedical applications of nanotechnology giving great promise for improving disease prevention, diagnosis, treatment and in particular tissue regeneration (Murthy, 2007).

Since natural human tissues include nano-scale subcellular and extracellular components, artificial nanomaterials mimic the scales of tissue components (Zhang \& Webster, 2009). Cells make contact with other cells and with the extracellular matrix with membrnes that have nanoscale features. It has been shown that nanomaterials, with their biomimetic features, can accelerate the rate of cell growth and proliferation and promote tissue acceptance due to reduced immune response (Oh et al., 2009). One of the most useful properties of nanomaterials, which have been extensively investigated, is their ability to interact with proteins that control cell functions. This may make nanomaterials very useful, and perhaps even necessary, tools for regenerating various tissues such as those of the bone, cartilage, blood vessels and nervous system (Liu \& Webster, 2007).

Although a series of technological improvements in tissue regeneration have been acheived using conventional methods, a variety of problems still faces current implants. Nanotechnology could provide several solutions to these problems. A wide range of nanomaterials have been made from organic and inorganic composites, just like conventional materials. However, nanotehnology has the ability to control material properties more closely by assembling components at the nanoscale. These nanomaterials (nanoparticles, nanotubes, nanofibers, nanoclusters, nanocrystals, nanowires, nanorods and 
nanofilms) can be fabricated by multiple and available nanotechnologies. Electrospining, self assembly, phase separation, photolithography, thin film deposition, chemical etching, chemical vapor deposition and electron beam lithography are all techniques currently used to synthesize nanomaterials with ordered or random nanotopographies (Chen \& Ma, 2004).

Conventional tissue replacement, using allografts and autografts, cannot satisfy high performance demands and improvements are necessary. Nanotechnology has been used to fabricate cytocompatible biomimetic nanomaterials that provide biological substitutes useful in restoring and improving tissue functions. Moreover, 2-dimensional tissue cell culture systems on flat glass, coated petri dishes or plastic substrates cannot simulate the natural tissue microenvironments. Normal tissue cells are located in a complex network of 3 dimensional extracellular matrix with nanoscale fibers. Nanomaterials could be fabricated that accurately simulate the dimensions and architecture of natural human tissue, allowing significantly improved performance of the cultured cells (Gelain et al., 2006). The composition and topography of a tissue engineered material could even produce cellenvironment interactions that determine the implant fate. Nanomaterials need to be designed to be biocompatible and to function without interrupting other physiological processes. In principle, they can promote normal cell growth and differentiation without any adverse tissue reaction. These nanomaterials must be biodegradable either to be removed via degradation or absorption to leave only native tissue. In addition, nanomaterials used in tissue regeneration should possess biomimetic features that allow cells to react normally to internal and external stimuli and to exchange the signals between those cells and the external environment.

This chapter reviews recent progress in the synthesis of nanomaterials for improving stem cell behavior and tissue regeneration. In addition, it highlights potentially valuable applications of nanotechnology in specific tissue regeneration.

\section{Effects of nanomaterials on stem cell behaviour and development of tissue regeneration}

Nanotechnology is an extremely promising advancement in synthetic methodologies used to functionalize nanomaterials with biomolecules. Nanomolecules could be modified to desired sizes, shapes, compositions and properties producing different types applied in tissue regeneration such as nanoparticles, nanosurfaces and nanoscaffolds.

\subsection{Nanoparticles}

Several studies have investigated the influences of different types of nanoparticles on the behaviour of stem cells applied in tissue regeneration. The effects of mesoporous silica nanoparticles conjugated with fluorescein isothiocyanate on human bone marrow mesenchymal stem cells has been investigated by several researchers. Internalization of silica nanoparticles into stem cells is mediated by both clathrin and actin-dependent endocytosis. Once inside the cell, the nanoparticles escaped the endolysosomal vesicles and did not affect stem cell viability or proliferation. They enhanced actin polymerization in mesenchymal stem cells. Moreover, regular osteogenic differentiation was successfully induced in the mesenchymal stem cells after the uptake of mesoporous silica nanoparticles in highly 
chondrogenic synovium (Huang et al., 2008 \& Shi et al., 2009). Fibrin polylactide caprolactone nanoparticles have been designed to induce chondrogenic differentiation in mesenchymal stem cells. These complex nanoparticles facilitated the upregulation of chondrogenesis marker genes. In addition, they effectively sustained chondrogenic differentiation and enhanced chondral extracellular matrix deposition by human adipogenic stem cells. Fibrin polylactide caprolactone nanoparticle complexes could be effectively used for in situ cartilage tissue regeneration from human stem cells (Jung et al., 2009).

The application of nanotechnology to stem cell biology might help to maximize therapeutic benefits and minimize possible undesired effects of stem cell therapy, through delivery of sufficient stem cells to the regions of interest with the smallest number of cells to untargeted regions. Tracking the fate, distribution, proliferation, differentiation of engulfed stem cells employed in tissue regeneration is essential to understand the mechanisms of participation of the cells in tissue repair. Nanotechnology can improve several techniques that would enable non-invasive detection of transplanted stem cells within the desired organs. Iron oxide nanoparticles are inorganic nanoparticles that can be synthesized easily in large quantities and different sizes using simple methods. Several studies reported that when iron oxide nanoparticles bind to the external cell membrane, they do not affect cell viability, although they may detach from the cell membrane or interfere with cell surface interactions (Bulte \& Kraitchman, 2004). Superparamagnetic iron oxide nanoparticles are successfully internalized via endocytosis in human mesenchymal stem cells. After their uptake, they are located inside cytoplasmic vesicles. Then, they are transferred to lysosomes in which degradation of the nanoparticles occurs, releasing free iron into the cytoplasm (Jing et al., 2008). Coating the surface of iron oxide nanoparticles modifies the surface of the particles for efficient uptake with minimum side effects on the cells. Coating superparamagnetic iron oxide nanoparticles with dextran improves their stability and solubility and prevents their aggregation. Another example of coating the surface of nanoparticles is provided by coating the superparamagnetic iron oxide nanoparticles with gold. The gold provides an inert shell around the nanoparticles and protects them from rapid dissolution within cytoplasmic endosomes and enhances magnetic resonance imaging (MRI) contrast. It has been shown, however, that dissolved iron oxide nanoparticles may produce free hydroxyl radicals which increase the rate of apoptosis and alterations in cellular metabolism (Emerit et al., 2001).

Concerning the effects of iron oxide nanoparticles on stem cell behaviour, magnetite iron oxide cationic liposomes can be applied efficiently to mesenchymal stem cell techniques. Mesenchymal stem cells incubated in osteogenic medium with these nanoparticles changed their shape from fibroblastic to polygonal, formed calcium nodules and increased in number five-fold compared with controls (Ito et al., 2004). In addition, superparamagnetic iron oxide nanoparticles have been shown to enhance the survival rate of stem cells up to $99 \%$, indicating that these nanoparticles improve stem cell viability (Delcroix et al., 2009). Moreover, superparamagnetic iron oxide nanoparticles did not influence the morphology, cell cycle, telomerase activity, proliferation or differentiation ability of labelled neural stem cells (Kea et al., 2009). Superparamagnetic iron oxide nanoparticles have been successfully applied to tracking the fate of several types of stem cells. For example, the migration of embryonic stem cells and bone marrow mesenchymal stem cells labelled with iron oxide nanoparticles towards a lesion site has been tracked using MRI. This labelling technique offers high resolution, speed, easy access and 3-dimensional capabilities and provides 
information not only for the transplanted cells, but also for the surrounding tissues, reporting edema or inflammation that may affect the fate of the grafted cells and reduce the recovery of damaged tissue (Sykova \& Jendelova, 2007). Another example is tracking human mesenchymal stem cells labelled with superparamagnetic iron oxide nanoparticles after transplantation for articular cartilage repair using MRI (Au et al., 2009). These observations have demonstrated the ability of using iron oxide nanoparticles to be useful in monitoring and tracking the fate of transplanted stem cells apparently without affecting their behaviour, although, selection of the type and concentration of nanoparticles is critically important.

In addition to iron oxide nanoparticles, quantum dots have been much used for cell tracking in in tissue regeneration. Quantum dots are fluorescent semiconducting nanocrystals that overcome the limitations of conventional labelling methods. Several researchers have studied the application of quantum dots to monitoring physiological changes inside living cells by labelling the intracellular organelles or specific proteins with quantum dots. They could monitor cellular migration, track cell lineage and investigate stem cell behaviour. Quantum dots can bind to individual molecules on the cell surface and serve in tracking the motion of those molecules. For example, quantum dots have been applied to demonstrate changes in integrin dynamics during osteogenic differentiation of human bone marrow cells (Chen et al., 2007). Numerous studies have demonstrated a variety of techniques of the cellular uptake of quantum dots. These nanoparticles can be delivered into cells by microinjection, endocytosis, liposome-mediated transfection and special peptide delivery (Chang et al., 2008). Delivered quantum dots were found to escaping lysosomal degradation at the beginning of the uptake. Thereafter, lysosome expression was enhanced and all cellular quantum dots were shown in lysosome vesicles. After the uptake of quantum dots, into several types of stem cells, such as mesenchymal stem cells, cytoskeletal reorganization took place. This action revealed the formation of wide and flat leading lamellipodia filled with a dense actin network (Chang et al., 2009). Human mesenchymal stem cells labelled with quantum dots represented the same viability comparing with the unlabelled human mesenchymal stem cells from the same subpopulation (Shah et al., 2007), suggesting that quantum dots could be used safely for long term labelling of stem cells. Moreover, embryonic stem cells could be labelled with quantum dots for cellular tracking in vivo without affecting the viability, proliferation or differentiation of the embryonic stem cells (Lin et al., 2007). These studies demonstrated that quantum dots could enable cellular and molecular imaging and tracking the fate of stem and progenitor cells used in tissue regeneration with high sensitivity and high spatial resolution. These applications are supported by an extensive number of advanced imaging techniques, giving a great impact on tissue regeneration studies.

\subsection{Nanosurfaces}

Mammalian cells are surrounded by nanostructures formed by biomolecules arranged geometrically in different configurations. These arrangements affect cell behaviour by producing chemical signals such as growth factors or physical signals such as tensile forces caused by interactions with the surrounding nanostructured extracellular matrix. Nanotechnology provides nanotopographical surfaces that can guide cellular adhesion, spreading, morphology, proliferation and differentiation. Cells react differently according to 
the nanotopography of their environment, which influences their cytoskeletal organization, attachment, and migration. Nanofabrication techniques provide several types of nanosurfaces for tissue regeneration.

Nanosurfaces of different materials with structural modification, such as the presence of large, medium and small nanoscale grooves, pores, pits, ridges and nodules can be recognized by cultured cells. A wide range of cell types, such as fibroblasts (Dalby et al., 2003b), osteoblasts (Lenhert et al., 2005) and mesenchymal stem cells (Biggs et al., 2008), are influenced by nanoscale grooves with dimensions that mimic those in vivo. Cellular morphology depends on cell type and on groove depth and width. Mesenchymal stem cells seeded on nanogrooves respond by aligning their shape and elongation in the direction of the grooves (Dalby et al., 2003b). Human osteoblasts cultured on ordered nanoscale groove/ridge arrays, fabricated by photolithography, were affected significantly (Biggs et al., 2008). The authors seeded human osteoblasts on grooves of $330 \mathrm{~nm}$ depth and different widths $(10,25$ and $100 \mu \mathrm{m}$ in width). They concluded that adhesion formation was not affected in $100 \mu \mathrm{m}$ wide groove/ridge arrays, although upregulation of genes involved in skeletal development was induced. In addition, increased osteospecific functions were observed. $25 \mu \mathrm{m}$ wide grooves/ ridges were shown to be associated with a reduction in supermature adhesions and an increase in focal complex formation. However, that osteoblast adhesion was significantly reduced in $10 \mu \mathrm{m}$ wide groove/ridge arrays. Moreover, grooves manufactured on nanosurfaces promoted the elongation and nuclear polarization in the cultured cells (Charest et al., 2004). Cell membranes stopped at the largest grooves but bridged over the narrowest and deepest ones (Matsuzaka et al., 2003). Electron beam lithography has also been used to generate nanoscale patterns for culturing mesenchymal stem cells (Dalby, 2009). The patterns ranged from highly ordered through controlled disorder, to total randomness. The authors concluded that nanoscale change in surface topography altered mesenchymal stem cell differentiation. Successful osteoconversion of the cultured cells using $\pm 50 \mathrm{~nm}$ level of disorder was demonstrated. The cells focal adhesions interacted with the material surface and affected by several signalling pathways, such as $G$ protein and cytoskeletal signalling. These signalling factors modulated cell sensing, morphology, contractility, proliferation and differentiation. Altering the nanotopography of the surface material influenced the cytoskeletal arrangements (Curtis et al., 2006). Mechanical changes were transmitted from the cytoskeleton to the nucleus, affecting the genomic expression patterns and cell phenotype (Dalby et al., 2007).

Among hard carbon coatings, nanocrystalline diamond has been applied successfully to cultured osteogenic and endothelial cells. Nanocrystalline diamond possesses promising electrical and optical properties, high hardness, low friction coefficient and good compatibility (Bacakova et al., 2007). Nanocrystalline diamond has been used in the form of films to improve the mechanical and physical properties of body implants. In addition, it has been shown to attract cell colonization, its surface nanostructure simulating the architecture of extracellular matrix molecules. Nanocrystalline diamond layers deposited on silicon substrates improves the adhesion and growth of osteogenic and endothelial cells (Grausova et al., 2008). The authors concluded that these nanostructured surfaces gave good support for cellular viability and proliferation and could be applied usefully in tissue regeneration. Furthermore, ultrasmooth nanostructured diamond has been used in 
orthopaedic implants. Several studies were performed on this material, the authors describing their surface modification techniques and cytocompatability (Clem et al., 2008). The studies demonstrated that hydrogen-terminated ultrasmooth nanostructured diamond surfaces supported robust mesenchymal stem cell adhesion and survival. However oxygen and fluorine terminated surfaces resisted cell adhesion. It was concluded that chemical and physical modifications of ultrasmooth nanostructured diamond could promote or prevent cell/biomaterial interactions. Moreover, mesenchymal stem cell adhesion and proliferation were significantly improved on ultrasmooth nanostructured diamond compared with the commonly used and biocompatible cobalt-chrome. There was also osteoblastic differentiation and deposition of mineralized matrix in mesenchymal stem cells. Ultrasmooth nanostructured diamond was found to reduce debris particle release from orthopaedic implants without influencing osseointegration.

Controllable self-assembly of nanonodules has been demonstrated to occur during chemical depositioning of materials on specifically conditioned microtopographical surfaces (Ogawa et al., 2008). The substrate could be a nonmetalic material such a as biodegradable polymer. The biological potential of the nanonodular surfaces affecting the behaviour of cultured cells using titanium dioxide has been investigated (Kubo et al., 2009). Titanium as a substrate material was proven to be non cytotoxic and was applied in therapeutic and implantable devices used in tissue regeneration. Micro-nano-hybrid surfaces, consisting of nanoscale nodules within microscale pits, were created by applying nanonodular self assembly techniques. These surfaces mimicked the biomineralized matrices with greater surface area and roughness. Changing the assembly time controlled the size of the nanonodules. The addition of nanonodules of different sizes $(100-300-500 \mathrm{~nm})$ to micropits selectively promoted osteoblast functions. In addition, these nanonodular topographies enhanced osteoblastic proliferation and differentiation. These advantages were 3 times greater in the nanonodules with a diameter of $300 \mathrm{~nm}$ within the micropits, when implanted in a rat femur model. Cell spread was enhanced on the micro- nano-hybrid surfaces. After 3 hours incubation, osteoblasts were shown to be larger and their cell processes and cytoskeletons started to develop on the nanonodular surfaces, while they remained small and circular on the micropit surface alone. Meanwhile, marked cytoplasmic localization of the focal adhesion protein vinculin was shown on the micro-nano-hybrid surfaces, compared with those on the micropit surface which had faint expression.

Another application of titanium in tissue regeneration is the use of nanocrystalline titanium surfaces. This type of nanometer surface roughness promotes osteoblasted adhesion. This nanosurface enhances cell growth and demonstrates extensive wear resistance due to high hardness and strength (Wang \& Li, 2003). Cell compatability studies on nanosized titanium particles showed enhanced osteoblast function and largeer deposition of calcium minerals (Webster et al., 2000). One of the most effective nanostructured titanium surfaces for enhancing the attachment, proliferation and spreading of mesenchymal stem cells is layerby-layer assembled titanium dioxide nanoparticle thin films. This technique depends on electrostatic attraction between oppositely charged species such as titanium dioxide nanoparticles. The advantage of layer-by-layer assembly is that the adsorption of material cn be controlled with nanometer precision. Titanium dioxide thin films have been proved to be an optimal surface for rapid attachment and spreading of cells (Kommireddy et al., 2005). Increasing the number of layers in titanium dioxide thin films has been shown to increase 
surface roughness. Higher numbers of attached cells were observed on 4-layer titanium dioxide thin film than on a 1-layer thin film, with a faster rate of spreading on the rougher surface (Kommireddy et al., 2006). Moreover, multilayered and functionalized titanium films composed of chitosan and plasmid DNA demonstrated significant high transfection efficiency in mesenchymal stem cells (Hu et al., 2009). The authors reported high production levels of alkaline phosphatase and osteocalcin. They concluded that multilayered titanium films with chitosan and plasmid DNA promoted the differentiation of osteoprogenitor cells into mature osteoblasts over long time.

\subsection{Nanoscaffolds}

Nanotechnology provides the tissue regeneration field with nanostructures that might accurately simulate the natural 3-dimensional microenvironment of cells. This approach provides a complex network of nanoscale fibers and extracellular ligands, such as many types of collagens, laminin and fibronectin, that are poorly reproduced in the conventional 2-dimensional systems. Growth of cells in 2-dimensional cultures has been shown to reduce the production of particular extracellular matrix proteins, with consequent morphological changes and increase in spreading. The advancement in the technology of nanostructures enhances the scope of fabricating 3-dimensional nanoscaffolds that could potentially mimic the architecture of natural human tissue. These nanostructured scaffolds could control and direct cellular behaviour and interactions with the extracellular matrix. Scaffolds have been designed in the form of nanofibers, nanotubes, nanowires, nanorods, nanocrystals and nanofilms. These nanostructured scaffolds with their biomimetic features and excellent physicochemical properties, stimulated cellular adhesion, growth, morphology, proliferation, altered gene expression and promoted cellular differentiation. The structural features of these nanoscaffolds were engineered according to the nature of cell response which was desired. The scaffolds were designed in a manner that provided a surface to promote cell attachment, spreading and growth while encouraging the formation of a porous network that offered a suitable path for nutrient transmission and tissue ingrowth (Chen \& Ma, 2004). These novel nanoscaffolds had excellent mechanical properties that offered structural support until the new tissue would be formed, as they degraded at a rate matching the new tissue formation and provided substrate for cell migration and survival. They were biocompatible and the products of their degradation were also biocompatible (Smith et al., 2010). These nanostructured scaffolds provided the functional role of the native extracellular matrix with growth factors that regulated the cell fate and bioactive peptide sequences that could bind receptors and activate intracellular signalling pathways (Boudreau \& Jones, 1999).

Several techniques have been designed for the fabrication of nanofibrous scaffolds to be employed in tissue regeneration. Electrospinning techniques have been the most commonly used. An electric field is applied to draw a polymer solution from an orifice to a collector, producing polymer fibers with diameters ranging in size from $50 \mathrm{~nm}$ to several microns. These resulted lengths mimicked that of native collagen fibrils (Baker et al., 2009). Several types of synthetic and natural biomaterials have been used to form nanofibrous scaffolds such as poly (caprolactone) (PCL), poly (lactic-co-glycolic acid) (PLGA) poly (L-lactic acid) (PLLA), collagen, gelatine and fibrinogen; molecules that have been applied extensively in 
tissue regeneration. Another technique for nanofibrous fabrication is self-assembly. Molecular self-assembly has been applied to produce supramolecular architectures (Silva et al., 2004). This technique produces nanofiber diameters much smaller than those produced using electrospinning. Molecular self-assembly has been less effective in producing macropores for mass transport and cell accommodation. Phase separation techniques have also been also employed to fabricate nanofibers with diameters ranging from $50-500 \mathrm{~nm}$ and much higher surface -to-volume ratios than produced by other techniques (Chen et al., 2006).

\section{Applications of nanotechnology in specific tissue regeneration}

Recent studies have been conducted on the promises and applications of nanotechnology in the regeneration of specific tissues, such as bone, cartilage, vascular and neural tissues.

\subsection{Bone and cartilage regeneration}

Various types of traumatic bone and cartilage damage - bone fractures, osteoarthritis, osteoporosis or bone tumours - represent common and significant clinical problems. However, the treatment of such problems with traditional implant materials only lasts 10 15 years on average and implant failures originating from implant loosening, inflammation, infection, osteolysis and wear debris frequently occur. There is a very urgent need to develop a new generation of cytocompatible bone and cartilage substitutes to regenerate bone and cartilage tissues at diseased sites that could last the life time of the patient (Zhang \& Webster, 2009).

Bone is effectively a nanocomposite that consists of a protein-based soft hydrogel template formed of collagen, non-collagenous proteins such as laminin, fibronectin and vitronectin, water, and hard inorganic components such as hydroxyapatite, calcium and phosphate. Specifically, $70 \%$ of the bone matrix is composed of nanocrystalline hydroxyapatite which is typically $20-80 \mathrm{~nm}$ long and 2-5 nm thick. Nanostructured bone extracellular matrix closely surrounds and affects adhesion, proliferation and differentiation of mesenchymal stem cells, osteoblasts, osteoclasts and fibroblasts. Moreover, cartilage is a poorly regenerating tissue composed of a small percentage of chondrocytes but dense nanostructured extracellular matrix rich in collagen fibers, proteoglycans and elastin fibers. The limited regenerative properties of cartilage originate from a lack of chondrocyte mobility in the dense extracellular matrix as well as an absence of progenitor cells and the vascular network necessary for efficient tissue repair (Vasita \& Katti, 2006). Development of nanotechnology might provide clinical medicine with new prospects in bone and cartilage reconstruction. Nanotechnology employs engineered materials with the smallest functional organization called nanomaterials that are able to interact with biological systems at a nanoscale (El-Sadik et al., 2010). Nanomaterials could be grown or self-assembled to stimulate the dimensions of natural entities, such as collagen fibers. After decreasing material size into nanoscale, dramatically increased surface area, surface roughness and surface area to volume ratios could be created, leading to superior physiochemical properties such as mechanical, electrical, optical, catalytic, magnetic properties. These biomimetic features with the nanostructured extracellular matrix of bone and cartilage played a key role in stimulating cell growth as well as guided tissue regeneration (Jang et al., 2009). Numerous researchers 
fabricated cytocompatible biomimetic nanomaterial scaffolds encapsulating cells, such as stem cells, chondrocytes and osteoblasts. In addition, to the dimensional similarity to bone/cartilage tissue, nanomaterials also exhibited unique surface properties, such as surface topography, surface chemistry, surface wettabilty and surface energy, due to their significantly increased surface area and roughness compared to conventional or micron structured materials. As is known, material surface properties mediate specific protein adsorption and bioactivity, such as fibronectin, vitronectin and laminin, before cells adhere on implants, further, they regulate cell behaviour and dictate tissue regeneration. Furthermore, an important criterion for designing orthopaedic implant materials is the formation of sufficient osseointegration between synthetic materials and bone tissue. Studies have demonstrated that nanostructured materials with cell-favourable surface properties could promote greater amounts of specific protein interactions to more efficiently stimulate new bone growth compared to conventional materials (Webster et al., 2001). This is one of the underlying reasons that nanomaterials are superior to conventional materials for bone growth. Therefore, by controlling surface properties, various nanophase ceramic, polymer, metal and composite scaffolds have been designed for bone/cartilage tissue engineering applications (Zhang \& Webster, 2009).

There have been significant advances in the development of bone scaffolds with various compositions and 3 dimensional configurations using a variety of techniques such as the electrospining process for the fabrication of nanofibrous matrices. Several studies have reported the performance of nanofibrous materials in guiding cells to initially adhere to, and spread over, the nanostructures, as well as triggering them to secrete appropriate extracellular matrix molecules targeted to the bone and cartilage tissues. The boneassociated cells and the progenitor/stem cells showed initial responses which were anchorage-dependant. The nanofibrous substratum provided favourable conditions for cell anchorage and growth. Further osteoblastic differentiation and mineralization have also been reported to be regulated in a positive manner on nanofibrous surfaces (Woo et al., 2007). One particular requirement of bone tissue regeneration was that the scaffold should be porous, to incorporate large number of cells. The 3-dimensional scaffolds provided the necessary support for bone cells to attach, grow and differentiate and defined the overall shape of a bone tissue cultured transplant (Jang et al., 2009). Nanofibrous and nanotubular scaffolds were fabricated to mimic collagen fibers in bone and cartilage. Natural collagen is a triple helix self assembled into nanofibers of $300 \mathrm{~nm}$ in length and $1.5 \mathrm{~nm}$ in diameter. A new nanofiber composite was designed with the same self-assembly pattern as collagen and hydroxyapatite crystals in bone by directly nucleating and aligning the hydroxyapatite on the long axis of a nanofiber. Mesenchymal stem cell behaviour on self-assembled peptide amphiphile nanofiber scaffolds was investigated. Significantly enhanced osteogenic differentiation of mesenchymal stem cells was recorded in the 3-dimensional scaffolds compared to 2-dimensional static conventional tissue cultures.

Other types of nanofibers used in bone regeneration include the natural polymers. Natural polymeric nanofibers, such as poly(caprolactone) (PCL), poly(lactic-co-glycolic acid) (PLGA) poly(L-lactic acid) (PLLA), collagen, gelatine and fibrinogen, are excellent candidates for bone and cartilage tissue engineering applications. These biomaterials possess properties that are useful for bone regeneration, such as biodegradability, flexibility, shape availability and ease of fabrication. Nanoporous polymer matrices can be fabricated via electrospinning, phase 
separation, particulate leaching, chemical etching and 3 dimensional printing techniques (Zhang \& Webster, 2009). Poly(caprolactone) (PCL) was first suggested to be a degradable nanofiber matrix for bone regeneration, and it demonstrated good support of the rat bone marrow stromal cells and in vitro matrix formation at 4 weeks, including collagen I and calcium phosphate (Yoshimoto et al., 2003). A cell-nanofiber construct was implanted in rat omenta for 4 weeks (Shin et al., 2004). It revealed the formation of collagen I and mineralization similar to bone like extracellular matrix, highlighting its usefulness in bone tissue regeneration. A combination of degradable polymeric nanofibers with bioactive inorganic metals was proved to enhance osteogenic differentiation and calcification of bone matrix. The inorganic phase improved the biological properties of polymers in the bone forming process. Gelatin-hydroxyapatite nanofibers was fabricated (Kim et al., 2005). Hydroxyapatite nanocrystals were distributed in the gelatin matrix and produced an organized hybrid matrix. This composite enhanced osteoblastic differentiation and could be applied usefully in dentistry. In a similar way, collagen-hydroxyapatite (Song et al., 2008) and chitosan-hydroxyapatite (Zhang et al., 2008) nanofibers were generated mimicking the extracellular matrices.

An additional excellent choice of nanomaterials for the reconstruction of bone tissue was the bone-bioactive inorganics such as bioactive glass, ceramics and calcium phosphates. Silica based sol-gel glass mixed with a polymer binder was generated into a nanofibrous mesh by an electrospining technique. Fibers ranging from $84 \mathrm{~nm}$ to $640 \mathrm{~nm}$ in size were produced (Kim et al., 2006). The large surface area of the nanofibers, and the consequent ionic reaction with the surrounding medium, induced the formation of a bone mineral-like apatite phase on their surfaces. Osteogenic proliferation and differentiation of rat mesenchymal stem cells were found to be enhanced on the bioactive glass nanofiber substrates more than on conventional bioactive glass. Nanophase metals were investigated for orthopaedic tissue regeneration. They are characterized by the presence of more particle boundaries at their surfaces than the conventional micron metals. Linear patterns of nano-features of titanium were created via electron beam evaporation. These patterns induced greater osteoblast adhesion than the micron-rough regions and guided osteoblast morphology and alignment. Highly porous titanium dioxide nanotube layers were fabricated on titanium by anodization. Titanium was anodized electrochemically in dilute hydrofluoric acid electrolyte solutions to produce nanotubes with diameters of $100 \mathrm{~nm}$ and lengths of $500 \mathrm{~nm}$ into the titanium dioxide layers of titanium. Nanotubular anodized titanium greatly improved osteoblastic function and significantly increased chondrocytic adhesion, promoting bone and cartilage cellular growth (Zhang \& Webster, 2009).

\subsection{Vascular tissue regeneration}

Researchers have come a long way to develop vascular grafts of great efficacy to replace damaged blood vessels, using materials that produce minimal interactions with the inflowing blood and adjacent tissues. Nanomaterials have been found to improve vascular endothelial and smooth muscle functions. Aligned biodegradable poly(L-lactid-co-epsiloncaprolactone) PLLA-CL (75:25) nanofibrous scaffolds have been tested for their ability to fabricate tubular scaffolds for vessels. These nanofibers demonstrated the mechanical strength needed to sustain high pressure of the human circulatory system and the necessary properties that mimic the dimensions of natural extracellular matrix of human coronary 
artery. They provided an excellent architecture for endothelial and smooth muscle cell adhesion and proliferation. The aligned fibers affected the behaviour of the smooth muscle cells, and the cytoskeleton is organized to follow the direction of the nanofibers ( $\mathrm{Xu}$ et al., 2004). Electrospun nanofibers fabricated from natural polymers have been established to develop constructs for vascular tissue regeneration. Electrospun collagen and elastin nanofibers were shown to be good scaffolding systems for the engineering of artificial blood vessels (Boland et al., 2004). Another polymer that promoted the endothelial and vascular smooth muscle cell proliferation was the biodegradable poly(lactic-co-glycolic acid) (PLGA), which produced vascular grafts with nanometer surface features. These nanostructures enhanced fibronectin and vitronectin adsorption from serum leading to better vascular cell responses (Miller et al., 2007). Moreover, self-assembled peptides have been fabricated into scaffolds that mimic the vascular basement membrane with excellent cytocompatability. These peptide scaffolds promote endothelialisation and enhance nitric oxide release and laminin and collagen IV deposition by the endothelial cell monolayer (Genove et al., 2005). Titanium nanostructures have been reported to enhance vascular cell adhesion and proliferation greatly. Competitive endothelial cell functions were promoted over that of vascular smooth muscle cells, solving the problem of the overgrowth of smooth muscle cells in vascular stents (Choudhary et al., 2007).

\subsection{Neural tissue regeneration}

Nanostructure designs have been shown to promote the functional performance of neuronal cells and neural tissue repair. They possess the necessary cytocompatibilty properties for improved neuronal growth, mechanical properties that last long enough to physically support neural tissue regeneration, and electrical properties that stimulate and control neuron behaviour and guide neural tissue repair. Biodegradable and biocompatible novel nanofibers and nanotubes have been fabricated with controlled architecture and components and efficient topography; they promoted neural tissue regeneration. Nanofibrous poly (L-lactic acid) (PLLA) and poly (caprolactone) (PCL) scaffolds designed via electrospining and phase separation demonstrated significant cytocompatibility properties useful for neural tissue regeneration. Incorporation of laminin into the nanofibers created a biomimetic scaffolds for peripheral nerve repair as laminin is an extracellular protein that promotes neurite outgrowth (Koh et al., 2008). Another example for the addition of laminin onto the poly (L-lactic acid) (PLLA) nanofibers was investigated for the culture of the tissues of rat dorsal root ganglia (Patel et al., 2007). Cultures revealed significant longer neurite length more than those cultured on poly (L-lactic acid) (PLLA) nanofibers without laminin. These findings demonstrated the advantages of biosynthetic nanomaterials over the synthetic ones. Moreover, the topography of the electrospun nanofibers scaffolds affected the behaviour of the cultured dorsal root ganglia. Significant extension and elongation of neurites were shown on aligned fibers compared with cultured on randomly oriented nanofibers. The neurites grew in a radial manner on the aligned nanofibers. Those that grew in the direction of the fibers had a faster growth rate than the others indicating that the aligned nanofibrous scaffolds served in guiding neurite orientation and cell alignment (Chow et al., 2007).

Electrospun Chitosan on poly(caprolactone) (PCL) nanofibrous scaffolds provided excellent mechanical properties that enhanced Schwann cell proliferation (Zhang \& Webster, 2009). 
Chitosan micro and nanofiber mesh tubes have also been investigated for nerve reconstruction (Wang et al., 2008). The authors observed early recovery of sensory functions and elongation of the regenerating axons in $10 \mathrm{~mm}$ rat sciatic nerve gap after implantation of the nanofiber mesh tubes. Covalent binding of synthetic and natural materials have been demonstrated in the conjugation of collagen onto a copolymer of methyl methacrylate and acrylic acid electrospun nanofibers (Cao et al., 2009). Increased neurite length of cortical neural stem cells, in proportion to collagen content, was found, indicating that this combination improved the attachment and viability of the cultured neural stem cells. Peptide nanofibrous scaffolds fabricated by self-assembly induced favourable neural cell responses and enhanced neuronal cell functions, outgrowth and functional synapse formation (Zhang \& Webster, 2009). Other types of scaffolds are the carbon nanotubes and nanofibers. They were found to guide axon regeneration and improve neural activity as a result of good electrical conductivity, strong mechanical properties and their similar nanoscale dimensions to neurites. Multiwalled carbon nanotubes have been applied for the growth of neurons: a $200 \%$ increase in total neurite length and a $300 \%$ increase in the number of branches and neurites have been demonstrated. In addition, decreased astrocyte proliferation, and consequent decreased glial scar tissue formation, was shown on carbon nanofibers with a polymer composite. Moreover, it was found that astrocytes attached and proliferated less on carbon nanofibers with the smallest nanometer diameter and the highest surface energy (Mckenzie et al., 2004). Carbon nanofibers were shown to limit astrocyte functions, leading to decreased glial scar tissue formation which is essential for increased neuronal implant efficacy.

\section{Safety issues involved in the use of nanotechnology}

Despite the wide range of applications of nanotechnology in the tissue regeneration studies, still there is a lack of information concerning the influence of nanomaterials on human health. Data available for the safety of nanomaterials, particularly in the field of tissue regeneration, are limited and the mechanisms of their toxicity are still poorly understood. Several studies indicated that a small size, a large surface area and the ability to generate reactive oxygen species increase the potential of nanomaterials to induce cell injury. However, other studies have indicated that, for example, ceramic nanoparticles were safer to osteoblasts than conventional ceramic microparticles. On the other hand, cellular uptake of nanoparticles and their effects on the physiological processes of the cells and their organelles should be deeply investigated before such materials are applied to human tissues. It has been shown, for example, that degradation of nanomaterials used in artificially engineered joints produced toxic responses due to the use of heavy metals such as iron, nickel and cobalt catalysts (Zang \& Webster, 2009).

Recent researches in the field of tracking the engrafted stem cells have demonstrated that the safety of quantum dots depends on their physiochemical properties, dose and exposure. Cytotoxicity of quantum dots has been observed owing to the presence of heavy metals such as cadmium and selenium in their cores. Coating the core of quantum dots was recorded to effectively reduce their toxicity to a significant level. Several strategies have been applied to decrease the toxicity of quantum dots. Coating the core with a shell of zinc sulphide reduces the toxicity by blocking the oxidation of the core by 
air, making them biologically inert. Another technique uses large protein molecules such as bovine serum albumin to could slow the photo-oxidation of the core. Moreover, labelling quantum dots with biomolecules such as arginine-glycine-aspartic acid removed all the toxic effects on cultured stem cells (Solanki et al., 2008). It is recommended to study the appropriate properties and concentrations of different nanoparticles used in cultured and transplanted cells and their safety limits and to deeply understand the physicochemical, molecular and physiological processes of nanomaterials before introducing them into the human bodies.

\section{Conclusion}

Nanotechnology has shown great potential for numerous tissue regeneration applications. Nanomaterials have achieved one of the major challenges of tissue regeneration which is mimicking the architecture of natural extracellular matrix. Designed nanostructures such as nanoparticles, nanosurfaces and nanoscaffolds have been used to promote stem cell cultures which will speed up understanding, controlling and guiding tissue regeneration studies of different tissues, such as bone, cartilage, vascular and neural tissues. It is suggested that the creation of such nanostructures would advance greatly the field of tissue regeneration. However, nanomaterials require more testing and investigations before full use in human tissue repair. Further understanding of their interactions with biological systems is still needed.

\section{References}

Au, K.; Liao, S.; Lee, Y. et al. (2009). Effects of Iron Oxide Nanoparticles on Cardiac Differentiation of Embryonic Stem Cells. Biochem Biophys Res Commun, Vol.379, (2009), pp. 898-903

Bacakova, L.; Grausova, L.; Vacik, J. et al. (2007). Improved Adhesion and Growth of Human Osteoblast-Like MG 63 Cells on Biomaterials Modified with Carbon Nanoparticles. Diamond Relat Mater, Vol.16, No.12 (December 2007), pp. 2133-2140

Baker, B.M.; Handorf, A.M.; Ionescu, L.C.; Li, W. \& Mauck, R.L. (2009). New Directions in Nanofibrous Scaffolds for Soft Tissue Engineering and Regeneration. Expert Rev Med Devices, Vol.6, No.5, (September 2009), pp. 515-532

Biggs, M.J.P.; Richards, R.G.; McFarlane, S.; Wilkinson, C.D.W.; Oreffo, R.O.C. \& Dalby M. J. (2008). Adhesion Formation of Primary Human Osteoblasts and The Functional Response of Mesenchymal Stem Cells to 330? nm Deep Microgrooves. J R Soc Interface, Vol.5, (2008), pp. 1231-1242

Boland, E.D.; Matthews, J.A.; Pawlowski, K.J. et al. (2004). Electrospinning Collagen and Elastin: Preliminary Vascular Tissue Engineering. Front Biosci, Vol.9, (2004), pp. 1422-1432

Boudreau, N.J. \& Jones, P.L. (1999). Extracellular Matrix and Integrin Signalling: The Shape of Things to Come. Biochem J, Vol.339, No.3, (1999), pp. 481-488

Bulte, J.W.M. \& Kraitchman, D.L. (2004). Monitoring Cell Therapy Using Iron Oxide MR Contrast Agents. Curr Pharm Biotechnol, Vol.5, No.6, (2004), pp. 567-584 
Cao, H.; Liu, T. \& Chew, S.Y. (2009). The Application of Nanofibrous Scaffolds in Neural Tissue Engineering. Advanced Drug Delivery Reviews, Vol.61, No.12, (October 2009), pp. 1055-1064

Chang, J.; Su, H. \& Hsu, S. (2008). The Use of Peptide- Delivery to Protect Human AdiposeDerived Adult Stem Cells from Damage Caused by The Internalization of Quantum Dots. Biomaterials, Vol.29, (2008), pp. 925-936

Chang, J.; Hsu, S. \& Su, H. (2009). The Regulation of The Gap Junction of Human Mesenchymal Stem Cells Through The Internalization of Quantum Dots. Biomaterials, Vol.30, (2009), pp. 1937-1946

Charest, J.L.; Bryants, L.E.; Garcia, A.J. \& King, W.P. (2004). Hot Embossing for Micropatterned Cell Substrates. Biomaterials, Vol.25, (2004), pp. 4767-4775

Chen, V.J. \& Ma, P.X. (2004). Nano-Fibrous Poly(L-Lactic Acid) Scaffolds with Interconnected Spherical Macropores. Biomaterials, Vol.25, No.11, (2004), pp. 20652073

Chen, V.J.; Smith, L.A. \& Ma, P.X. (2006). Bone Regeneration on Computer-Designed NanoFibrous Scaffolds. Biomaterials, Vol.27, (2006), pp. 3973-3979

Chen, H.; Titushkin, I.; Stroscio, M. \& Cho, M. (2007). Altered Membrane Dynamics of Quantum Dot-Conjugated Integrins During Osteogenic Differentiation of Human Bone Marrow Derived Progenitor Cells. Biophys J, Vol.92, (2007), pp. 1399-1408

Choudhary, S.; Haberstroh, K.M. \& Webster, T.J. (2007). Enhanced Functions of Vascular Cells on Nanostructured Ti for Improved Stent Applications. Tissue Engineering, Vol.13, (2007), pp. 1421-1430

Chow, W.N.; Simpson, D.G.; Bigbee, J.W. \& Colello, R.J. (2007). Evaluating Neuronal and Glial Growth on Electrospun Polarized Matrices: Bridging the Gap in Percussive Spinal Cord Injuries. Neuron Glia Biol, Vol.3, (2007), pp. 119-126

Clem, W.C.; Chowdhury, S.; Catledge, S.A. et al., (2008). Mesenchymal Stem Cell Interaction with Ultra-Smooth Nanosrtuctured Diamond for Wear-Resistant Orthopaedic Implants. Biomaterials, Vol.29, No.24-25, (August-September 2008), pp. 3461-3468

Curtis, A.S.G.; Dalby, M.J. \& Gadegaard, N. (2006). Cell Signaling Arising from Nanotopography: Implications for Nanomedical Devices. Nanomedicine, Vol.1, (2006), pp. 67-72

Dalby, M.J.; Riehle, M.O.; Yarwood, S.J.; Wilkinson, C.D. \& Curtis, A.S. (2003b). Nucleus Alignment and Cell Signaling in Fibroblasts: Response to a Micro-Grooved Topography. Exp Cell Res, Vol.284, (2003b), pp. 274-282

Dalby, M.J.; Biggs, M. J.; Gadegaard, N.; Kalna, G.; Wilkinson, C.D. \& Curtis, A.S. (2007). Nanotopographical Stimulation of Mechanotransduction and Changes in Interphase Centromere Positioning. J Cell Biochem, Vol.100, (2007), pp. 326-338

Dalby, M.J. (2009). Nanostructured Surfaces: Cell Engineering and Cell Biology. Nanomedicine, Vol.4, No.3, (April 2009), pp. 247-248

Delcroix, G.J.; Jacquart, M.; Lemaire, L. et al., (2009). Mesenchymal and Neural Stem Cells Labelled with HEDP-coated SPIO Nanoparticles: In Vitro Characterization and Migration Potential in Rat Brain. Brain Res, Vol.1255, (2009), pp. 18-31 
El-Sadik, A.O.; El-Ansary, A. \& Sabry, S.M. (2010). Nanoparticle-Labeled Stem Cells: A Novel Therapeutic Vehicle. Clinical Pharmacology: Advances and Applications, Vol.2, (2010), pp. 9-16

Emerit, J.C.; Beaumount; C. \& Trivin, F. (2001). Iron Metabolism, Free Radicals, and Oxidative Injury. Biomed Pharmacother, Vol.55, No.6, (2001), pp. 333-339

Gao, J. \& Xu, B. (2009). Applications of Nanomaterials Inside Cells. Nanotoday, Vol.4, (2009), pp. 37-51

Gelain, F.; Bottai, D.; Vescovi, A. \& Zhang, S. (2006). Designer Self-Assembling Peptide Nanofiber Scaffolds for Adult Mouse Neural Stem Cell 3-Dimensional Cultures. PLoS ONE, Vol.1, No.1, (2006), e119

Genove, E.;Shen, C.; Zhang, S. \& Semino, C.E. (2005). The Effect of Functionalized SelfAssembling Peptide Scaffolds on Human Aortic Endothelial Cell Function. Biomaterials, Vol.26, No.16, (June 2005), pp. 3341-3351

Grausova, L.; Kromka, A.; Bacakova, L.; Potocky, S.; Vanecek, M. \& Lisa, V. (2008). Bone and Vascular Endothelial Cells in Cultures on Nanocrystalline Diamond Films. Diamond and Related Materials, Vol.17, No.7-10, (July-October 2008), pp. 1405-1409

$\mathrm{Hu}$, Y.; Cai, K.; Luo, Z. et al. (2009). Surface Mediated in Situ Differentiation of Mesenchymal Stem Cells on Gene-Functionalized Titanium Films Fabricated by Layer-by- Layer Technique. Biomaterials, Vol.30, No.21, (July 2009), pp. 3626-3635

Huang, D.; Chung, T.; Hung, Y. et al. (2008). Internalization of Mesoporous Silica Nanoparticles Induces Transient but not Sufficient Osteogenic Signals in Human Mesenchymal Stem Cells. Toxicol Appl Pharmacol, Vol.231, (2008), pp. 208-215

Ito, A.; Hibino, E.; Honda, H. et al. (2004). A New Methodology of Mesenchymal Stem Cell Expansion Using Magnetic Nanoparticle. Biomechemical and Engineering J, Vol.20, (2004), pp. 119-125

Jang, J.; Castano, O. \& Kim, H. (2009). Electrospun Materials as Potential Platforms for Bone Tissue Engineering. Advanced Delivery Reviews, Vol.61, (2009), pp. 1065-1083

Jing, X.; Yang, L.; Duan, X. et al. (2008). Invivo MR Imaging Tracking of Magnetic Iron Oxide Nanoparticle Labelled, Engineered, Autologus Bone Marrow Mesenchymal Stem Cells Following Intra-Articular Injection. Joint Bone Spine, Vol.75, (2008), pp. 432-438

Jung, Y.; Chung, Y.; Kim, S.H. et al. (2009). In situ Chondrogenic Differentiation of Human Adipose Tissue-Derived Stem Cells in a TGF-b1 Loaded Fibrin-Poly(LactideCaprolactone) Nanoparticulate. Biomaterials, Vol.30, (2009), pp. 4657-4664

Kea, Y.; Hu, C.; Jianga, X. et al. (2009). In vivo Magnetic Resonance Tracking of FeridexLabelled Bone MarroDerived Neural Stem Cells after Autologous Transplantation in Rhesus Monkey. J Neurosci Methods, Vol.179, (2009), pp. 45-50

Kim, H.W.; Song, J.H. \& Kim, H.E. (2005). Nanofiber Generation of Gelatin-Hydroxyapatite Biomimetics for Guided Tissue Regeneration. Adv Funct Matr, Vol.15, (2005), pp. 1988-1994

Kim, H.W.; Kim, H.E. \& Knowles, J.C. (2006). Production and Potential of Bioactive Glass Nanofibers as a Next-Generation Biomaterial. Adv Funct Mater, Vol.16, (2006), pp. 1529-1535 
Koh, H.S.; Yong, T.; Chan, C.K. \& Ramakrishna, S. (2008). Enhancement of Neurite Outgrowth Using Nanostructured Scaffolds Coupled with Laminin. Biomaterials, Vol.29, No.26, (September 2008), pp. 3574-3582

Kommireddy, D.S.; Ichinose, I.; Lvov, Y.M. \& Mills, D.K. (2005). Nanoparticle Thin Films: Surface Modification for Cell Attachment and Growth. J Biomed Nanotechnol, Vol.3, (2005), pp. 286-290

Kommireddy, D.S.; Sriram, S.M.; Lvov, Y.M. \& Mills, D.K. (2006). Stem Cell Attachment to Layer-by-Layer Assembled TiO2 Nanoparticle Thin Films. Biomaterials, Vol.27, No.24, (August 2006), pp. 4296-4303

Kubo, K.; Tsukimura, N.; Iwasa, F. et al. (2009). Cellular Behavior on TiO2 Nanonodular Structures in a Micro-to-Nanoscale Hierarchy Model. Biomaterials, Vol.30, No.29, (October 2009), pp. 5319-5329

Lenhert, S.; Meier, M.B.; Meyer, U.; Chi, L. \& Wiesmann, H.P. (2005). Osteoblast Alignment, Elongation and Migration on Grooved Polystrene Surfaces Patterned by LangmuirBlodgett Lithography. Biomaterials, Vol.26, (2004), pp. 563-570

Lin, S.; Xie, X. \& Patel, M.R. (2007). Quantum Dot Imaging for Embryonic Stem Cells. BMC Biotechnol, Vol.7, (2007), pp. 67

Liu, H. \& Webster, T.J. (2007). Nanomedicine for Implants: A Review of Studies and Necessary Experimental Tools. Biomaterials, Vol.28, No.2, (January 2007), pp. 354369

Mckenzie, J.L.; Waid, M.C.; Shi, R. \& Webster, T.J. (2004). Decreased Functions of Astrocytes on Carbon Nanofiber Materials. Biomaterials, Vol.25, No.7-8, (March-April 2004), pp. 1309-1317

Matsuzaka, K.; Walboomers, X.F.; Yoshinari, M.; Inoue, T. \& Jansen, J.A. (2003). The Attachment and Growth Behavior of Osteoblast-like Cells on Microtextured Surfaces. Biomaterials, Vol.24, (2003), pp. 2711-2719

Miller, D.C.; Haberstroh, K.M. \& Webster, T.J. (2007). PLGA Nanometer Surface Features Manipulate Fibronectin Interactions for Improved Vascular Cell Adhesion. J Biomed Mater Res A, Vol.81, No.3, (June 2007), pp. 678-684

Murthy, S.K. (2007). Nanoparticles in Modern Medicine: State of the Art and Future Challenges. International Journal of Nanomedicine, Vol.2, (2007), pp. 129-141

Ogawa, T.; Saruwatari, L.; Takeuchi, K.; Aita, H. \& Ohno, N. (2008). Ti Nano-Nodular Structuring for Bone Integration and Regeneration. J Dent Res, Vol.87, (2008), pp. 751-756

Oh, S.; Brammer, K.S.; Julie Li, Y.S.; et al. (2009). Stem Cell Fate Dictated Solely by Altered Nanotube Dimension. Proc Natl Acad Sci USA, Vol.106, No.7, (February 2009), pp. 2130-2135, ISSN 1729-8806

Patel, S.; Kurpinski, K.; Quigley, R. et al., (2007). Bioactive Nanofibers: Synergistic Effects of Nanotopography and Chemical Signaling on Cell Guidance. Nano Lett, Vol.7, (2007), pp. 2122-2128

Shah, B.S.; Clark, P.A.; Moioli, E.K.; Stroscio, M.A. \& Mao, J.J. (2007). Labelling of Mesenchymal Stem Cells by Bioconjugated Quantum Dots. Nano Lett, Vol.7, (2007), pp. 3071-3079 
Shi, X.; Wang, Y.; Varshney, R.R. et al., (2009). In-vitro Osteogenesis of Synovium Stem Cells Induced by Controlled Release of Bisphosphate Additives from Microspherical Mesoporous Silica Composite. Biomaterials, Vol.30, (2009), pp. 39964005

Shin, M.; Yoshimoto, H. \& Vacanti, J.P. (2004). In Vivo Bone Tissue Engineering Using Mesenchymal Stem Cells on a Novel Electrospun Nanofibrous Scaffold. Tissue Eng, Vol.10, (2004), pp. 33-41

Silva, G.A.; Czeisler, C.; Niece, K.L. et al. (2004). Selective Differentiation of Neural Progenitor Cells by High-Epitope Density Nanofibers. Science, Vol.303, (2004), pp. 1352-1355

Smith, I.O.; Liu, X.H.; Smith, L.A. \& Ma, P.X. (2010). Nano-Structured Polymer Scaffolds for Tissue Engineering and Regenerative Medicine. Wiley Interdiscip Rev Nanomed Nanobiotechnol, Vol.1, No.2, (March 2010), pp. 226-236

Song, J.H.; Kim, H.E. \& Kim, H.W. (2008). Electrospun Fibrous Web of Collagen-Apatite Precipitated Nanocomposite for Bone Regeneration. J Mater Sci Mater Med, Vol.19, (2008), pp. 2925-2932

Sykova, E. \& Jendelova, P. (2007). Migration, Fate and In vivo Imaging of Adult Stem Cells in the CNS. Cell Death Differ, Vol.14, (2007), pp. 1336-1342

Vasita, R. \& Katti, D.S. (2006). Nanofibers and Their Applications in Tissue Engineering. International Journal of Nanomedicine, Vol.1, No.1, (2006), pp. 15-30, ISSN 11769114

Wang, L. \& Li, D. Y. (2003). Mechanical, Electrochemical and Tribological Properties of Nanocrystalline Surface of Brass Produced by Sandblasting and Annealing. Surface and Coatings Technology, Vol.167, No.2-3, (April 2003), pp. 188-196

Wang, W.; Itoh, S.; Matsuda, A. et al. (2008). Influences of Mechanical Properties and Permeability on Chitosan Nano/Microfiber Mesh Tubes as a Scaffold for Nerve Regeneration. J Biomed Mater Res A, Vol.84, No.A, (2008), pp. 557-566

Webster, T.J.; Ergun, C.; Doremus, R.H.; Siegel, R.W. \& Bizios, R. (2000). Enhanced Functions of Osteoblasts on Nanophase Ceramics. Biomaterials, Vol.21, No.17, (September 2000), pp. 1803-1810

Webster, T.J.; Schadler, L.S.; Siegel, R.W. \& Bizios, R. (2001). Mechanisms of Enhanced Osteoblast Adhesion on Nanophase Alumina Involve Vitronectin. Tissue Engineering, Vol.7, No.3, (2001), pp. 291-301, ISSN 10763279

Woo, K.M.; Jun, J.H.; Chen, J.H.; et al. (2007). Nano-Fibrous Scaffolding Promotes Osteoblast Differentiation and Biomineralization. Biomaterials, Vol.28, (2007), pp. 335-343

Xu, C.Y.; Inai, R.; Kotaki, M. \& Ramakrishna, S. (2004). Aligned Biodegradable Nanofibrous Structure: A Potential Scaffold for Blood Vessel Engineering. Biomaterials, Vol.25, No.5, (February 2004), pp. 877-886

Yoshimoto, H.; Shin, H.; Terai, H. \& Vacanti, A. (2003). Biodegradable Nanofiber Scaffolds by Electrospinning and its Potential for Bone Tissue Engineering. Biomaterials, Vol.24, (2003), pp. 2077-2082

Zhang, Y.; Venugopal, J.R.; El-Turki, A.; Ramakrishna, S.; Su, B. \& Lim, C.T. (2008). Electrospun Biomimetic Nanocomposite Nanofibers of Hydroxyapatite/Chitosan for Bone Tissue Engineering. Biomaterials, Vol.29, (2008), pp. 4314-4322 
Zhang, L. \& Webster, T.J. (2009). Nanotechnology and Nanomaterials: Promises for Improved Tissue Regeneration. Nanotoday, Vol.4, No.1, (February 2009), pp. 66-80 


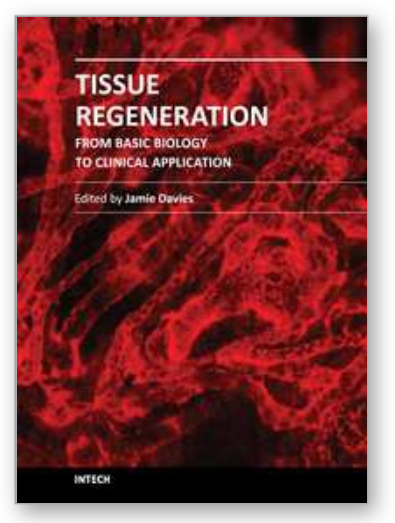

\author{
Tissue Regeneration - From Basic Biology to Clinical Application \\ Edited by Prof. Jamie Davies
}

ISBN 978-953-51-0387-5

Hard cover, 512 pages

Publisher InTech

Published online 30, March, 2012

Published in print edition March, 2012

When most types of human tissue are damaged, they repair themselves by forming a scar - a mechanically strong 'patch' that restores structural integrity to the tissue without restoring physiological function. Much better, for a patient, would be like-for-like replacement of damaged tissue with something functionally equivalent: there is currently an intense international research effort focused on this goal. This timely book addresses key topics in tissue regeneration in a sequence of linked chapters, each written by world experts; understanding normal healing; sources of, and methods of using, stem cells; construction and use of scaffolds; and modelling and assessment of regeneration. The book is intended for an audience consisting of advanced students, and research and medical professionals.

\title{
How to reference
}

In order to correctly reference this scholarly work, feel free to copy and paste the following:

Abir El-Sadik (2012). Novel Promises of Nanotechnology for Tissue Regeneration, Tissue Regeneration From Basic Biology to Clinical Application, Prof. Jamie Davies (Ed.), ISBN: 978-953-51-0387-5, InTech, Available from: http://www.intechopen.com/books/tissue-regeneration-from-basic-biology-to-clinicalapplication/novel-promises-of-nanotechnology-for-tissue-regeneration

\section{INTECH}

open science | open minds

\section{InTech Europe}

University Campus STeP Ri

Slavka Krautzeka 83/A

51000 Rijeka, Croatia

Phone: +385 (51) 770447

Fax: +385 (51) 686166

www.intechopen.com

\section{InTech China}

Unit 405, Office Block, Hotel Equatorial Shanghai

No.65, Yan An Road (West), Shanghai, 200040, China

中国上海市延安西路65号上海国际贵都大饭店办公楼 405 单元

Phone: +86-21-62489820

Fax: $+86-21-62489821$ 
(C) 2012 The Author(s). Licensee IntechOpen. This is an open access article distributed under the terms of the Creative Commons Attribution 3.0 License, which permits unrestricted use, distribution, and reproduction in any medium, provided the original work is properly cited. 